\title{
EUV Sprays: Jet-like eruptive activity on the solar limb
}

\author{
R. A. Harrison ${ }^{1}$, P. Bryans ${ }^{2}$, and R. Bingham ${ }^{1}$ \\ 1 Space Science and Technology Dept., Rutherford Appleton Laboratory, Chilton, Didcot, \\ Oxfordshire, OX11 0QX, UK \\ 2 Department of Physics and Applied Physics, University of Strathclyde, Glasgow, Scotland and Space Science \\ and Technology Dept., Rutherford Appleton Laboratory, Chilton, Didcot, Oxfordshire, OX11 0QX, UK
}

Received 28 May 2001 / Accepted 6 August 2001

\begin{abstract}
Jet-like eruptive events have been detected in the extreme ultraviolet (EUV) observations from the Solar and Heliospheric Observatory (SOHO) spacecraft. Some jet-like events are shown and for one event, that of January 22 2000, we provide a detailed analysis of the EUV observations. The January 22 observation was part of an on-going campaign to observe the onset of coronal mass ejections (CMEs) using extreme ultraviolet spectroscopy. A high-temperature fan-like jet was first detected in emission lines from plasmas at one million $\mathrm{K}$ and over, and it appears to be the onset of a narrow mass ejection identified later in coronagraph data. This was followed by a dramatic jet or finger of cool $(\leq 250000 \mathrm{~K})$, rotating plasma which extended vertically, high into the corona. It extended well beyond the field of view of the instrument and appears to relate to a small ejected cloud which is detected in subsequent coronagraph data. The spectroscopic characteristics are used to provide plasma diagnostic information for these two events, and this combined with images for a wide range of temperatures allows a thorough investigation of the processes leading to such eruptions. A simple accreting magnetic field model is used to descibe the basic features of the jet activity.
\end{abstract}

Key words. Sun: UV radiation - Sun: activity - Sun: atmosphere - Sun: corona

\section{Introduction}

One major goal in solar physics is to understand the onset processes of coronal mass ejections (CMEs). To this end, one substantial campaign of the SOHO (Solar and Heliospheric Observatory) mission has been the extreme ultraviolet (EUV), spectroscopic analysis of the onset phase of CMEs. An introduction to the CME-onset problem, to the campaign and to results for one observation sequence have been given by Harrison \& Lyons (2000). Further results from the campaign and related activities have been reported elsewhere (e.g. Innes et al. 1999; Harrison 1997).

The campaign consists of a limb-monitoring EUV sequence, using the SOHO Coronal Diagnostic Spectrometer (CDS) (Harrison et al. 1995) supported by synoptic observations from the SOHO Large Angle Spectroscopic Coronagraph (LASCO) (Brueckner et al. 1995), which monitors the overlying corona for CME events.

The eruptive events detected in the EUV with the CDS instrument include a class of jet-like phenomena, which are the EUV equivalent of $\mathrm{H} \alpha$ sprays. Figure 1 shows three examples of such ejecta taken in the light from the

Send offprint requests to: R. A. Harrison,

e-mail: Harrison@rl.ac.uk
O V emission line at $629 \AA$, which has a characteristic temperature of $250000 \mathrm{~K}$. The images are shown twice with differing intensity scales, to highlight different features. The top four panels of each frame show the event of 22 January 2000 (CDS observation run 18447). The images are taken at 16:09, 16:59, 17:49 and 18:38 UT, respectively. The principal jet-like event is seen in the lower portion of the 16:59 UT frame. It appears as a vertical, slightly non-radial jet extending beyond the field of view. It is not apparent in the 16:09 and 17:49 UT frames. The lighter images show the coincident appearance of a bright, small loop at the base of the jet. The darker images reveal that the jet extends to over 150 arcsec above the limb.

The middle four panels of the two frames show the event of 8 March 2000 (CDS observation run 18781). The images are taken at 21:01, 21:17, 21:33 and 21:49 UT (i.e. much faster cadence that the previous event). The beginnings of the jet are seen in the 21:17 UT image and the jet becomes brighter and extends well into the corona in the next frames. Though it is decaying in intensity by 21:49 UT, the darker images reveal that the jet extends to at least 160 arcsec in length. The lighter images reveal a compact bright patch directly under the jet on the solar surface. The resolution is not sufficient to show whether this is a loop or not. 

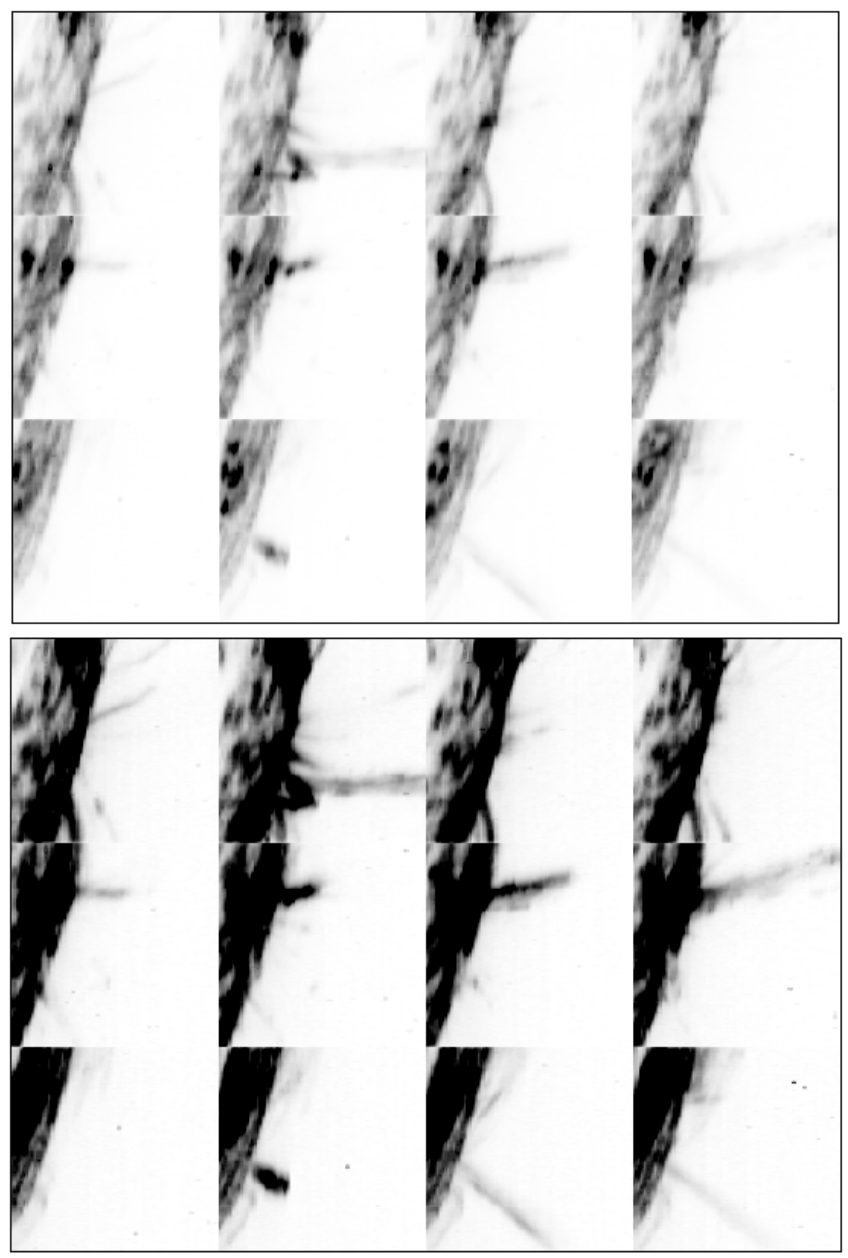

Fig. 1. Three examples of EUV jet-like events, on 22 January, 8 March and 19 March 2000, taken with the CDS instrument on SOHO. Each square frame is $4 \times 4$ arcmin square and the observation is displayed in the $250000 \mathrm{~K}$ emission line of $\mathrm{O} \mathrm{V}$ at $629 \AA$. Each row of four images shows a sequence from one event. The images are shown in negative form and repeated, one above the other, with two differing intensity scales. This highlights different intensity features and contrasts.

The lower four panels of the two frames show the event of 19 March 2000 (CDS observation run 18922). The images are taken at 12:20, 12:36, 12:52 and 13:08 UT. A blob or plasmoid-like feature shows the onset of the event in the 12:36 UT image which then extends at least 150 arcsec into the corona and dims in the 12:52 UT image. If the source of the event is this side of the limb, which appears to be the case, the footpoint projects, again, back to a compact, bright patch.

These three events display jet-like behaviour and have some common characteristics. The jets appear to be shortlived. The durations of the events are less than 1 hour $40 \mathrm{~min}$ for the 22 January event, about $30 \mathrm{~min}$ for the 8 March event, and less than $30 \mathrm{~min}$ for the 19 March event. The January event could be much shorter in duration, but the image cadence does not allow a better estimate. However, it seems that these jet-like eruptive events are of a highly transient nature.
In addition, the coincident location of bright features at the footpoints of the jets is worthy of note. For the January event, we can resolve the bright feature as a compact loop. It is not at all visible at times when the jet is not visible.

To explore the nature of this jet-like phenomenon, we present an analysis of the 22 January 2000 observations. We believe that these events are important for several reasons. For the 22 January ejections, we are witnessing the onset phase of an eruption later detected in the coronagraph field. Analysis of these events may be important for the understanding of mass ejection phenomena in general, and can certainly be used to compare the signatures of events detected in ultraviolet wavelengths in the low corona with white-light features detected in the outer corona.

In the next section, we describe the instrumentation and the details of the EUV observation sequence. This is followed by a description and interpretation of the 22 January events and a discussion of the importance of these observations for eruptive events in general.

\section{The EUV observation scheme}

The CDS instrument is a spectrometer operating in the EUV range from 150-800 A. It allows the detection and analysis of spectral emission lines from numerous trace ions in the solar atmosphere, with spatial, spectral and temporal resolving elements of down to 2 arcsec, $0.08 \AA$ and a few seconds, respectively (see Harrison et al. 1995 for a full description). The selected wavelength region contains emission lines which come from ions with characteristic temperatures in the range $20000 \mathrm{~K}$ to over 2 million $\mathrm{K}$, thus making this particular wavelength region extremely valuable for studies of the solar atmosphere where a wide temperature range exists.

The particular observing sequence used here has been used frequently as part of a CME onset campaign, described as Joint Observing Programme (JOP) 67. In this particular case, observations were made on the solar south-western limb between 15:03 UT and 23:19 UT on the 22 nd of January 2000 . The object of the sequence is to monitor a large area of the limb region using a range of emission lines representing a large range of temperatures.

Images some $240 \times 240$ arcsec in size are built up in all selected emission lines through a rastering process which is described in detail by Harrison \& Lyons (2000). A 4 arcsec by 240 arcsec slit is rastered across a 240 arcsec field. The exposure time of each slit position is $10 \mathrm{~s}$ and the full rastered image takes $951 \mathrm{~s}$. For this particular CME watch programme, in order to monitor a large region of the solar limb, the $240 \times 240$ arcsec images are produced in sets of mosaics, using three images at a time (see Fig. 3), i.e. three $240 \times 240$ arcsec frames are used to cover a large area of the limb and this mosaic is repeated over a period of many hours. The three images are directed at (centre of the image) 1009 arcsec west of Sun centre by $49 \operatorname{arcsec}$ 


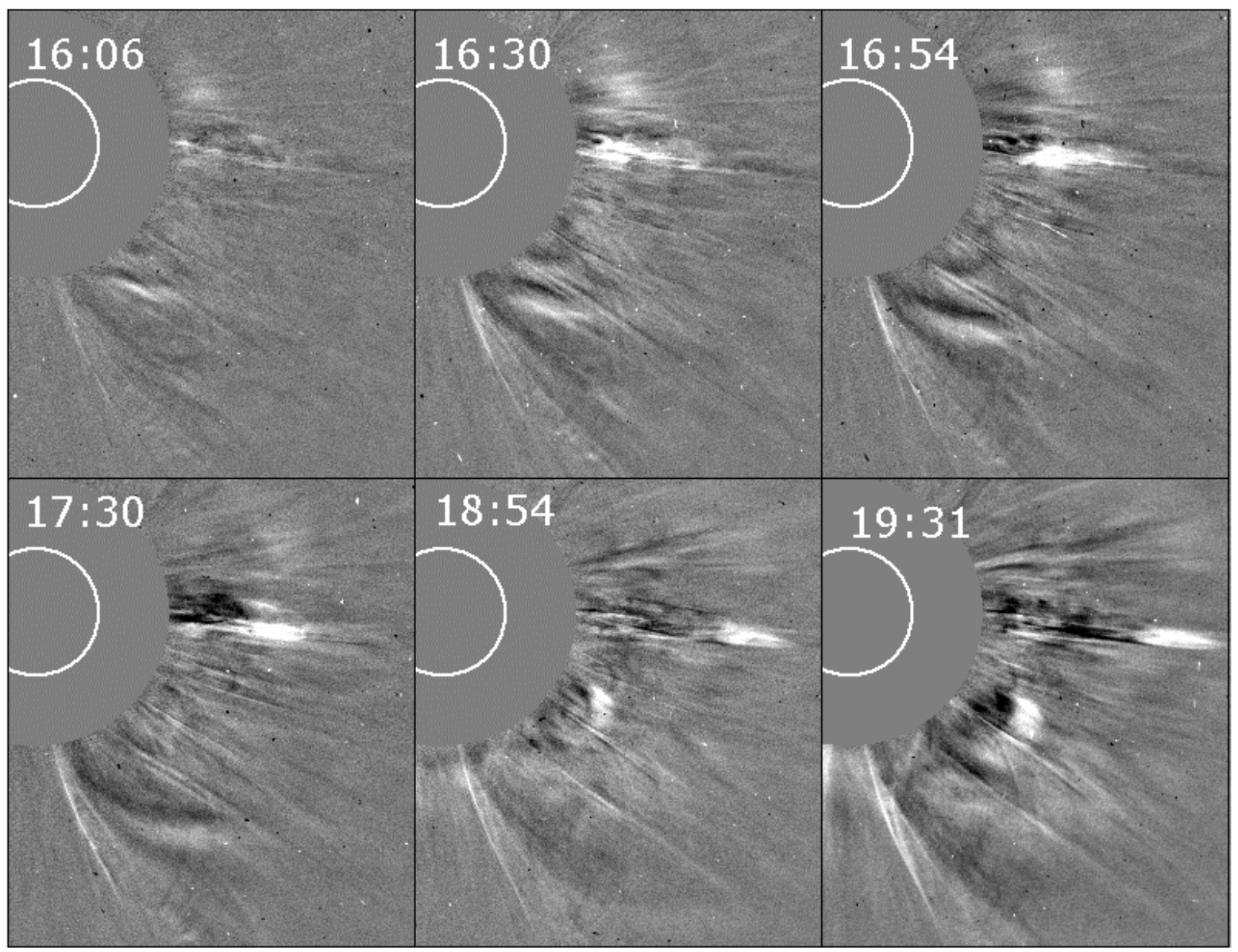

Fig. 2. White light C2 LASCO coronagraph images of the January 22 events. The time of each frame is given in UT. The location of the solar disc is indicated by the white circle. The images are displayed using a running differenced technique and, thus, white areas show density increase and dark areas show density depletion relative to each previous frame.

south of Sun centre, 979 arcsec west by 243 arcsec south and 928 arcsec west by 430 arcsec south.

The sequence was run for a period of 8 hours $50 \mathrm{~min}$ allowing 33 frames to be made, resulting in 11 mosaic images. The first image was taken from 15:03 UT and the last began at 23:19 UT. For each image, we returned data for 6 selected emission lines, as listed in Table 1. The line spectra are divided into 15 bins across the range shown. The number of returned lines was a play-off against the cadence, which even for this number of lines was 50 min. The selected lines were necessarily bright (to enable short exposures and, thus, fast rastering), and represent a wide range of temperatures, from $2000 \mathrm{~K}$ to 2 million $\mathrm{K}$. Two of the lines (the $\mathrm{Si} \mathrm{X}$ pair) provide a measure of density for million $\mathrm{K}$ plasmas when their intensity ratios are considered.

\section{White light coronagraph observations}

The LASCO coronagraph normally performs regular synoptic observations covering the full 360 degree corona with the differing coronagraph components at a rate which is adequate for this activity. Thus, no special operation was run. The events of January 22 are summarised by the
Table 1. Emission lines used for this study.

\begin{tabular}{lcr}
\hline Ion & Wavelength Range $\AA$ & Temperature K \\
\hline He I 584.33 & $583.5-585.2$ & 20000 \\
O V 629.73 & $629.0-630.6$ & 250000 \\
Mg IX 368.06 & $367.6-368.6$ & 1000000 \\
Si X 347.40 & $347.1-348.1$ & 1300000 \\
Si X 356.04 & $355.6-356.6$ & 1300000 \\
Fe XVI 360.76 & $360.3-361.3$ & 2000000 \\
\hline
\end{tabular}

selection of LASCO images shown in Fig. 2. These images are from the $\mathrm{C} 2$ coronagraph of LASCO, which has a field of view from 1.5 to 6 solar radii. The images are shown in a running differenced technique where each image is shown as a difference from the previous image. In this way, we highlight the coronal density enhancements as white regions and the depletions as dark regions.

The first image, taken at 16:06 UT shows the quiet pre-event corona. In the second frame, at 16:30 UT, a jetlike mass ejection has emerged from under the occulting disc just south of the western equator. From this point we will refer to this feature as the "jet". This jet continues to cross the corona in the subsequent images, reaching the 

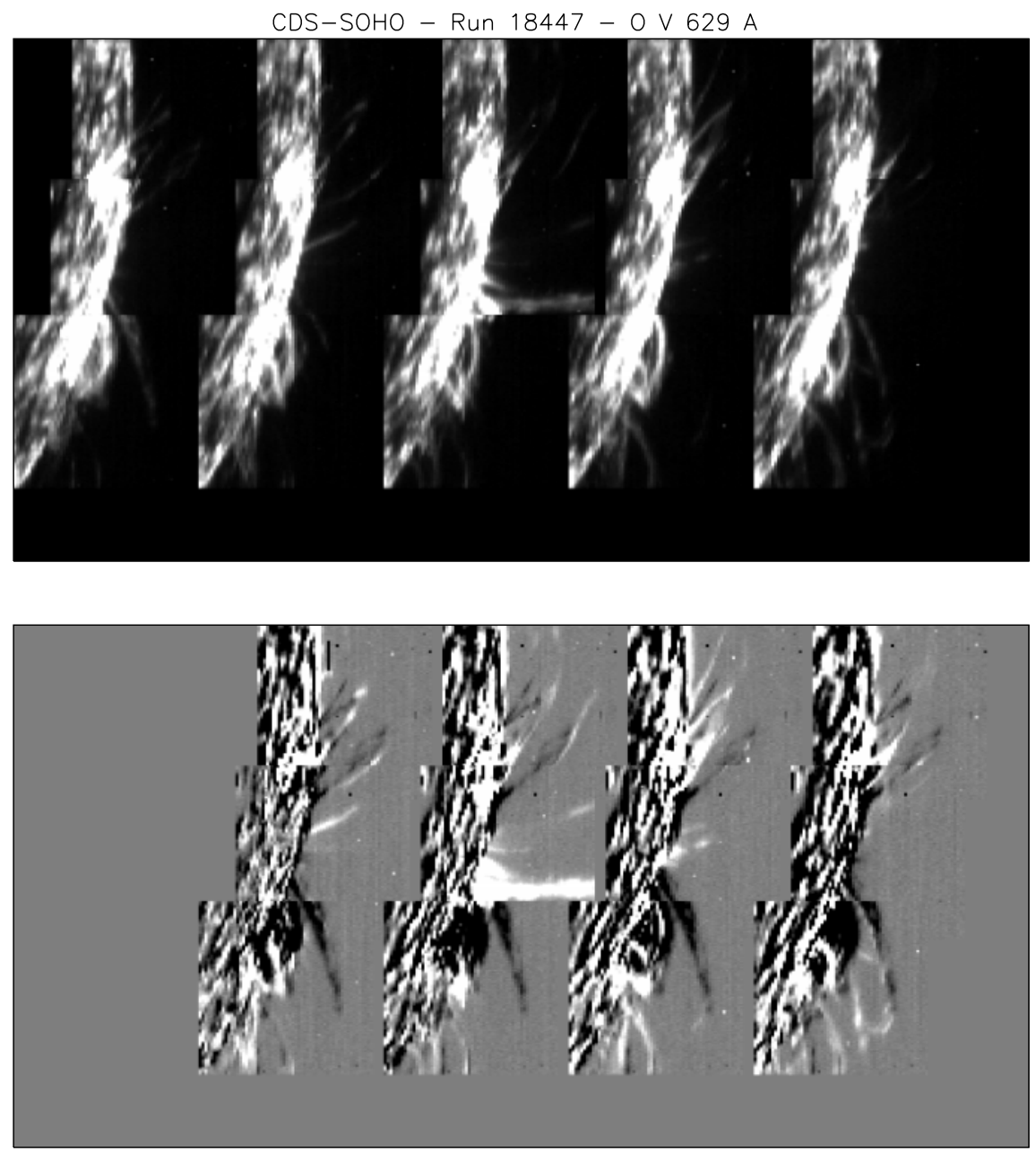

Fig. 3. The sequence of images on the south western solar limb taken in the O V emission line. Time runs from left to right with the first image starting at 15:03 UT and a 50 min cadence. The bottom row shows the same images with the first frame subtracted from each.

edge of the field of view at about 20:00 UT. The position angle of the jet is 267 degrees (measured eastwards from north), i.e. it is three degrees south of the western equator.

In the last two frames, a second bright event is detected as a small, bright cloud immediately above the southwestern limb. This feature was not evident in the 17:30 UT frame. It appears to be loop-like, with the brightest portion being the northern extreme of the loop. It appears to be followed by a small cavity. We will refer to this feature as the cloud. It appears to occupy position angles 225 to 245 degrees, though the southern extreme is difficult to determine.

These two bright features, the jet and cloud, may only be components of a much larger eruption. The bright rays and the curved coronal features extending into the corona from position angle 193 degrees, just west of the southern polar region, look remarkably like the southern extreme of a large, but poorly seen CME. Indeed, the curved feature appears to be whipping back (in particular between the 16:54 UT and 17:30 UT frames) as though it is part of an ascending loop. The jet event may be at the northern extreme, or northern leg, of this event, with the small cloud ejection in the south west being part of the central structure of the event.

By projecting the path of the jet back to the solar disc, an estimate of the onset time of this event was obtained. The accuracy of this method is somewhat crude; it is difficult to be certain that the same part of the jet is being measured from image to image. Such a projection does, however, give a reasonable estimate of a time range within which the jet is likely to have taken off.

A constant-speed fit to the data gives a speed of about $180 \mathrm{~km} \mathrm{~s}^{-1}$ with an onset time (from the limb and assuming no acceleration under the disc) of 13:45 UT. However, the time height profile of the jet does suggest a modest deceleration which, when fitted to the data, suggests an onset at 15:04 UT. Given the diffuse nature of the jet and the uncertainty of the time-height profile under the occulting disc, the uncertainty of the onset times must be of order \pm 0.5 hours at least.

The cloud is first seen in the LASCO frame at 18:30 UT. The time-height curve of this feature suggests an onset at about 16:30 UT \pm 0.5 hours. 

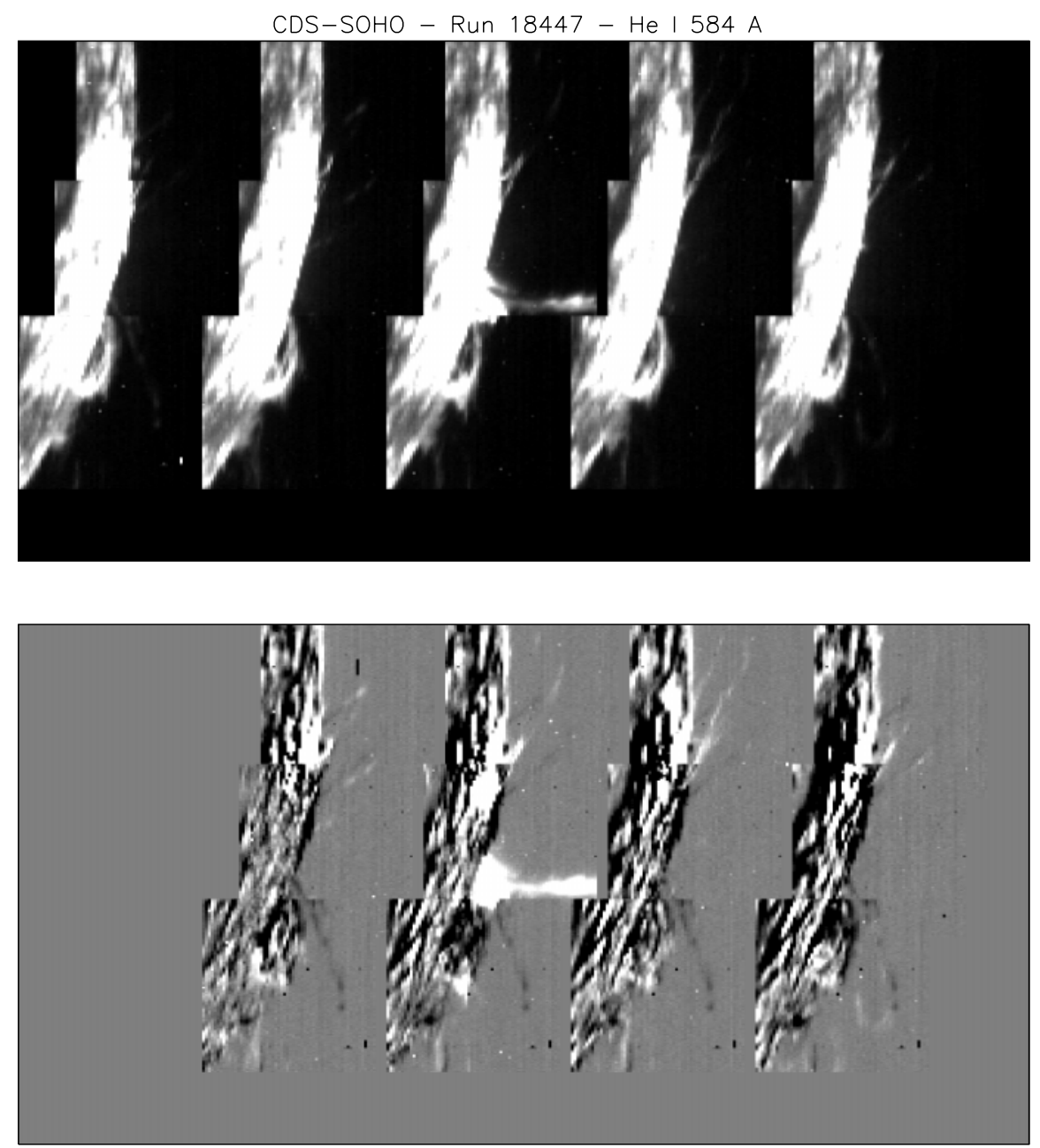

Fig. 4. The first five frames taken in the He I $584 \AA 20000 \mathrm{~K}$ line, showing the raw intensity data (top line) and the images with the first frame subtracted (bottom line) to highlight the changes with time.

\section{EUV observations}

The field of view of the EUV mosiac images covers the south-western limb in the area of active regions 8837 and 8824. Region 8837 was centred on position angle 265 degrees, just Earthward of the limb, and region 8824 was just beyond the limb at position angle 257 degrees. Both are well within the CDS field of view.

Figure 3 shows the first 5 of the 11 CDS mosaic frames taken during the sequence of January 22 (where each image is a mosaic of three $240 \times 240$ arcsec frames) for the $\mathrm{O} \mathrm{V}$ $629 \AA$ emission line. The details on the disc are slightly saturated in order to highlight the activity and structure above the limb. The $\mathrm{O} \mathrm{V}$ data are representative of temperatures of about $250000 \mathrm{~K}$, which we think of traditionally as transition region temperatures. The lower frames show the same data with the first frame subtracted from each, to highlight the changes with time. White reveals excess emission, and black decreased emission with respect to the first frame.

What these images reveal is a complex and highly variable system of loops occupying the south western limb. Most of the structures appear to be brightenings in closed loops which either extend southwards or northwards of the centre of the observed field. It is as if the region of interest is a confluence of magnetic systems, i.e. loop systems to the north and south share the region as a common footpoint location. However, one feature stands out as being of a highly transient nature and appears to show matter being ejected into the corona. This feature is the fingerlike vertical jet of plasma in the centre of the third image. This is from a raster taken at 16:59 UT, and it was the event shown in Fig. 1. The same region was imaged at 16:09 UT and 17:48 UT (in the mosaic frames on either side) and there was no sign of the ejection. The vertical jet is located at position angle 252 degrees but its path is non-radial. It would cross the lower limit of the LASCO field of view at considerably higher position angles.

The nature of this EUV jet can be seen further in the He I sequence of Fig. 4. The EUV jet can clearly be identified in the raw He I data, which is characteristic of temperatures up to $20000 \mathrm{~K}$. In the bottom row of the figure, again, we use image differencing to highlight changes with time. The EUV jet is striking in this display. This differenced sequence also stresses that the two frames preceding and following the EUV jet (in the He I and O V 



Fig. 5. A similar dispay to Fig. 3 but for the 1 million $\mathrm{K} \mathrm{Mg}$ IX emission line at $368 \AA$.

data) show almost no change at all from the first frame in the region of the jet, i.e. it is a highly transient event with no lasting change in the coronal features at these temperatures.

Similar displays to Figs. 3 and 4 are shown in Figs. 5 and 6 for the hotter ions of Mg IX and Fe XVI, which show plasmas at 1 million and 2 million $\mathrm{K}$.

The Mg IX raw data appear to show very little change with time yet the differenced images clearly show a bright fan of hot material, first detected at 16:09 UT. Since the event in the 16:09 UT differenced frame is bright, we can assume that it started later than the time of the first raster over that part of the Sun, i.e. at 15:19 UT. There is also a dark lane in the corona in the Mg IX data, north of the bright fan. The fan itself appears to originate a little north of the EUV jet seen in the $\mathrm{O} \mathrm{V}$ and He I data, i.e. at about 255 degrees. It is also highly non-radial. In the $\mathrm{Mg}$ IX data, there is no sign of the EUV jet, which is seen in the $\mathrm{O} \mathrm{V}$ and He I data.

The Fe XVI raw data also reveal little, but the differenced images show significant changes. The region where the Mg IX fan occurs is seen as a relatively dark area, with bright Fe XVI emission on either side, i.e. the Fe XVI images appear to reveal a two-prongued eruptive event, the brightest portions of which lie on either side of the Mg IX fan.

Using the EUV spectral information, so far we have looked at the most basic temperature information, over a range from 20000 to $2000000 \mathrm{~K}$ for these events. We now consider velocity information provided by the Doppler analysis of the data.

Figure 7 shows the raw $\mathrm{O} \mathrm{V}$ image at the time of the EUV jet. It shows the central $4 \times 4$ arcmin portion of the third CDS mosaic. The image is plotted using different colour tables to Fig. 3 to show more features on the disc and limb region. The EUV jet is clearly rooted in an area of some complexity with the bright, yet small loop adjacent to the jet, which was shown in Fig. 1, and evidence for other loop features. The right hand image of the figure shows a Doppler map of the same data. It indicates quite clearly that the EUV jet is a rotating column of plasma with the plasma on the southern side moving Earthwards and the northern side moving away from Earth. The rotational structure of the event is coherent from its root at the limb to the edge of the field. This event is reminiscent of the tornado-like events reported by Pike \& Mason (1998), but in this observation we are witnessing a rotating 

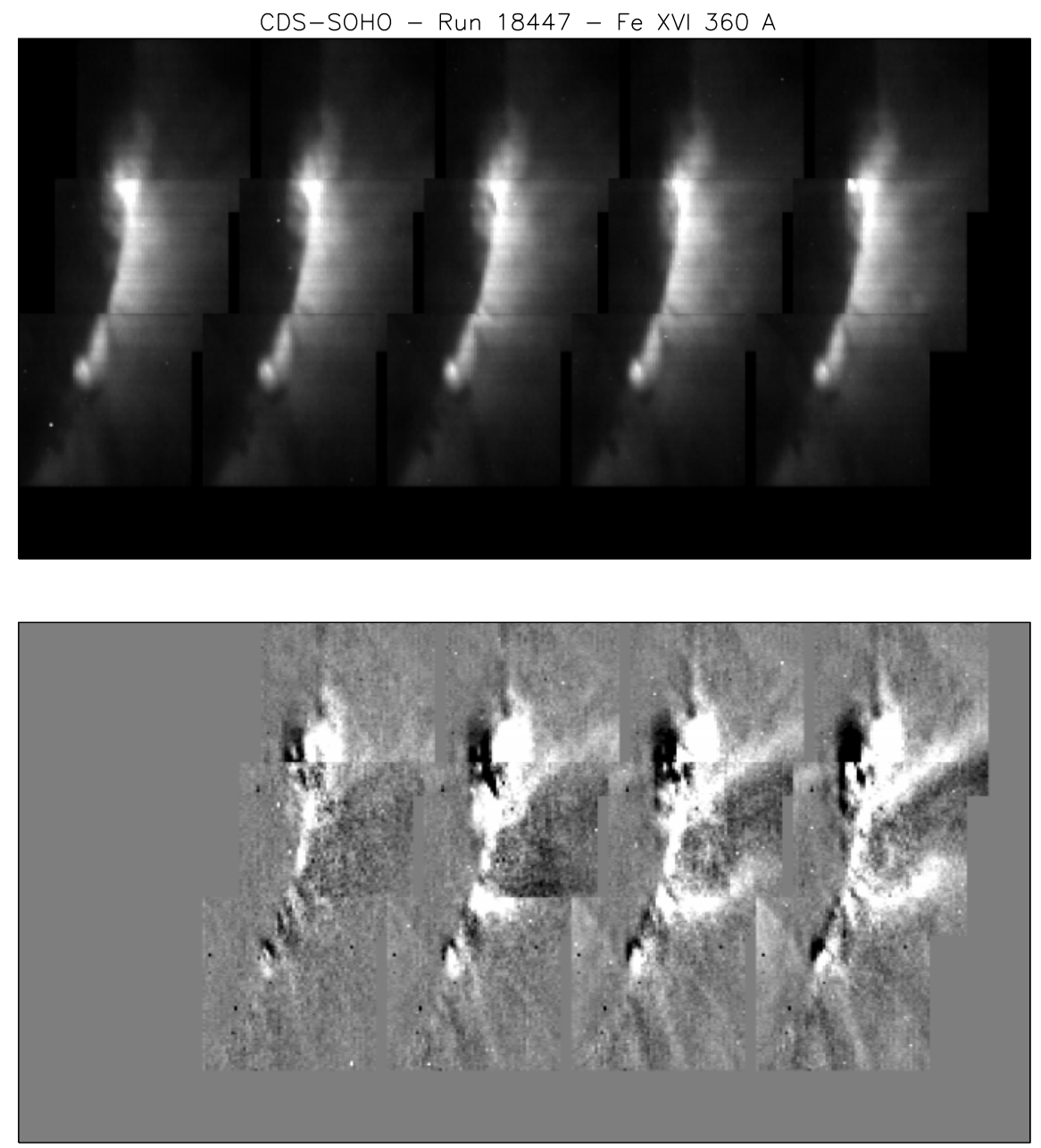

Fig. 6. A similar display to Fig. 3 but for the 2 million K Fe XVI emission line at $360 \AA$.

column rooted in an active region near the equator, rather than a polar coronal hole region.

Figure 8 shows the velocity characteristics of the EUV jet. Each CDS image, or pixel array, contains $120 \times$ 143 pixels. If we label the bottom left pixel as $(0,0)$ the centre-line of the jet runs almost perfectly along the line $y=42$. We have divided the pixels along the jet into boxes which are used to study the velocity profile of the jet. The jet is rooted in the disc at about pixel $(60,42)$, using the above notation. Thus, we take the six ten-pixel divisions in $x$, from 60 to 119 and for each we consider the blue shifted side (pixels $y=30-40$ ) and red shifted side (4353). Thus, we are considering 12 groups of 110 pixels to analyse the velocity structure of the jet.

For each pixel group we sum the emission from the $\mathrm{O} \mathrm{V}$ spectral window and fit a spectral line. The centre of the fitted line is noted and is plotted in Fig. 8. The blueshifted material is shown with asterisks and the red as crosses. The emission line is collected with 15 bins across the line. The ordinate is the location of the line-centre in pixels or bins in wavelength space. The centre of the fitted line falls between pixel 7.5 and 9.0. The wavelength conversion will be discussed later. The abscissa shows the six 110-pixel bins in order from the lowest (bin 1) to the highest (bin 6), so this is, in effect, altitude.

We show the data in this rather raw state to highlight the trends and to avoid any distraction from any other factors such as calibration, and errors in the altitude from any out of the plane of the sky property of the event.

If the plasma was showing an acceleration with altitude one would expect the red-shifted data to display an increasing peak location with altitude (or bin). One would expect also, a decreasing peak location with altitude (or bin) for the blue-shifted material. This is indeed the case. The solid line is a rough guide for the trend in the red-shifted data, showing an increase of $11 \mathrm{~km} \mathrm{~s}^{-1}$ per pixel with altitude. The dashed line is a rough guide for the trend in the blue-shifted data, showing an increase of $4 \mathrm{~km} \mathrm{~s}^{-1}$ per pixel with altitude. Clearly, these are not consistent. Both the red and blue shifted data show acceleration with altitude, but they ought to show the same value, for a consistent acceleration. However, although the trends are clear in these data, the errors on the emission line fitting is of order several tenths of a pixel and the best we could really state is that there is clear evidence for acceleration with altitude at a rate of about $8 \pm 4 \mathrm{~km} \mathrm{~s}^{-1}$ per pixel. 




Fig. 7. The left hand frame shows the O V raw image at 16:59 UT. For the same data, the right hand frame shows a Doppler map with blue and red denoting blue and red shifted material.

The absolute value of the velocity is best determined against the value at the disc, i.e. where the two lines in Fig. 8 cross. If we assume a zero shift at that point (with the peak located in pixel 7.9 to 8.0$)$ then we are seeing speeds of approximately $380 \mathrm{~km} \mathrm{~s}^{-1}$ (red) and $190 \mathrm{~km} \mathrm{~s}^{-1}$ (blue) where the plasma reaches the outer edge of the CDS field of view.

Figure 7 shows evidence for a small EUV loop at the root of the EUV jet, which is bright in the $\mathrm{O} V$ data. It is interesting to note that the Doppler image does not show a coherent velocity pattern for that loop; it is not easy to identify in the Doppler image. One can see mottled blue and red shifted material elsewhere in the image but it is the EUV jet that dominates the velocity structure.

A similar Doppler analysis of the fan-like Mg IX jet shows no sign of spiralling plasma motion.

Before continuing the spectral analysis, we briefly inspect the co-incident coronal imaging from the Extreme Ultraviolet Imaging Telescope (EIT) also aboard SOHO (Delaboudiniere et al. 1995). Figure 9 shows four images from a series of images taken in the Fe XII $195 \AA$ emission line at 1.6 million $\mathrm{K}$. These images are differenced from the previous frames, to highlight enhancements (white) and depletions (dark) in intensity. The south western limb region observed by CDS is shown. The first image, taken at 15:00 UT is coincident in time with the first of the mosaic images of CDS (Figs. 3 to 6). The image shows a bright fan-like feature with a dark loop to the north. This is very similar to the CDS Mg IX observation, which is slightly lower in temperature, i.e. the fan-like event referred

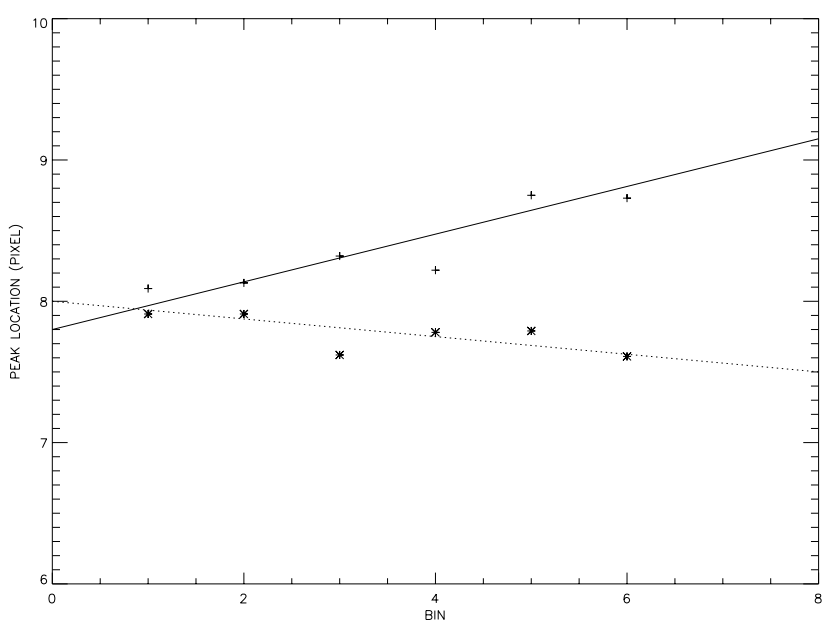

Fig. 8. The velocity profiles along the EUV jet. For the pixel groups described in the text the position of the peak of the fitted line is shown for the blue side of the jet (asterisks) and the red side of the jet (crosses), as a function of altitude. The abscissa bin number corresponds to altitude, as described in the text, with the photosphere to the left. The ordinate is the location of the peak of the fitted line, i.e. the bins correspond to pixels in wavelength space. The two lines show curves that would show an increase of $11 \mathrm{~km} \mathrm{~s}^{-1}$ per pixel in altitude in the red wing (solid line) and an increase of $4 \mathrm{~km} \mathrm{~s}^{-1}$ per pixel in altitude in the blue wing (dotted line).

to earlier is clearly identified in the EIT data. As noted before, it is not seen in the cooler lines. 

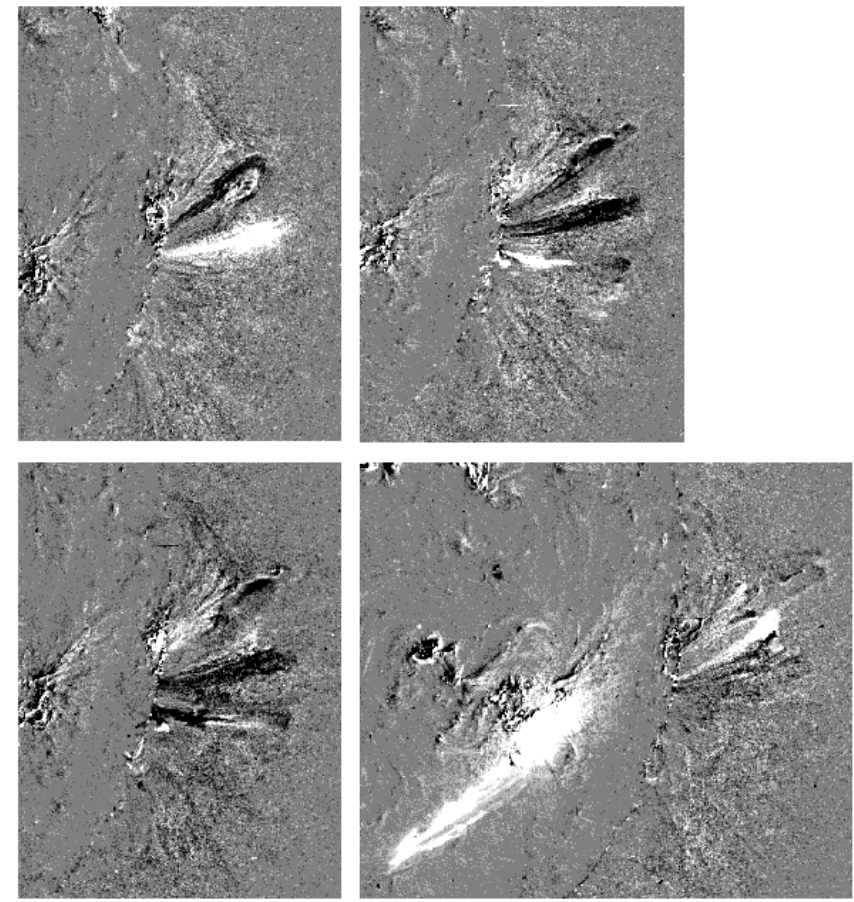

Fig. 9. Four differenced images from the SOHO/EIT instrument in the $195 \AA \mathrm{Fe}$ XII emission line directed at the same region on the south western limb on January 22, 2000. The images are taken at 15:00, 16:48, 17:00 and 18:00 UT (top left to bottom right).

The CDS EUV jet was detected at 16:59 UT and was clearly visible in the cooler, $\mathrm{O} \mathrm{V}$ and $\mathrm{He} \mathrm{I}$ lines. It was not detected in the Mg IX and Fe XVI emission. The EIT images at 16:48 UT and 17:00 UT span the onset time of that jet. Even though the EIT $195 \AA$ emission line is from an ion at 1.6 million $\mathrm{K}$, there is evidence for a small, bright jet, especially in the 16:48 UT image. The earlier fan-like event has faded in these frames and is seen as a dark lane. The small jet-like event is to the south. Its location is entirely consistent with the CDS jet, but the evidence from the CDS data would suggest that it should not be visible in the Fe XII image. It is possible that the EIT jet is actually emitted by a cooler line within the EIT bandpass, such as the O V line at 194.59 $\mathrm{A}$. However, in any case, there is evidence for a jet-like eruption, in support of the CDS data, and its location is consistent with the CDS interperation.

The last EIT image at 18:00 UT shows the corona after the jet-like event. It shows a significant brightening in one of the loops north of the jet site which is seen as a bright fan in the last of the $\mathrm{Mg}$ IX mosaic images of Fig. 5. However, perhaps of more importance, it shows a very significant brightening of an active region well onto the disc. This is an M1 class flare event. The coincident brightenings of the limb region and the flare may suggest that the two are connected. Such a large-scale connection would be consistent with the scale-size of the CME activity, but the CME eruptions described earlier had certainly occurred before 17:00 UT.
Let us now complete the CDS spectral analysis by considering the time-intensity behaviour of the main features detected in the 22 January data. There are three basic features, namely, the EUV jet seen in the $\mathrm{O} V$ data, the small, bright loop seen at the base of the EUV jet, and the hot, fan-like brightenings seen in the $\mathrm{Mg}$ and $\mathrm{Fe}$ data.

Using the same coordinate system as above, the looptop of the compact loop at the base of the EUV jet is contained within the pixel coordinates $X=43-52, Y=$ 25-43. The bulk of the EUV jet is contained within the area $X=85-110, Y=33-52$, and the base of the $\mathrm{Mg}$ "fan" is contained in the area $X=49-72, Y=66-93$.

Figure 10 shows the time-intensity curves for the different emission lines for the loop-top region as defined in the last paragraph. The Mg IX and Fe XVI lines show some gradual variation. The principal interest is in the period of the jet/fan activity, i.e. between 16:00 and 17:00 UT. The Mg IX intensity is gradually falling in this period and the Fe XVI intensity is gradually rising. This is suggestive of heating, but there is no indication of a sudden heating or cooling associated with the eruptive activity.

The $\mathrm{O} \mathrm{V}$ and He I data show the most dramatic activity in Fig. 10. For most of the period displayed, these lines show low intensity. For the image at 16:59 UT, both show a spike-like enhancement of intensity. This does not correspond to anything seen in the hotter lines. This is, of course, the brightening seen in the small loop in Figs. 1 and 7. In effect, the loop is just not visible except at the time when the jet is detected.

The two silicon lines used for this analysis are shown in Fig. 10 and these display similar intensity-time profiles, with the $356 \AA$ line more intense. Despite the larger error bars on these, due to the weaker intensities of these lines relative to the other lines in the study, the ratio does show a clear feature in the density history of the loop. The initial silicon intensity ratio is at the 1.1 level. According to Mason et al. (1997) this is representative of a density of $1.3 \times 10^{9} \mathrm{~cm}^{-3}$, for plasmas emitting in the region of $1.3 \times 10^{6} \mathrm{~K}$. However, there is a clear step up in the ratio, to about 1.55 at exactly the same time as the loop brightens dramatically in $\mathrm{O} \mathrm{V}$ and He I. The new density is of order $6.3 \times 10^{9} \mathrm{~cm}^{-3}$ (Mason et al. 1997), i.e. this suggests an almost 5 fold increase in density. However, this is rather anomalous; one would expect the Si X and Mg IX intensities to increase considerably in the face of such a density increase. In any case, the fact that the loop is impossible to locate in all images except $\mathrm{He} \mathrm{I}$ and $\mathrm{O} \mathrm{V}$ at the time of the jet-like eruption, it is quite possible that the Mg IX, Fe XVI and Si X emissions are not from the small loop at all, but are from hotter plasmas along the same line of sight.

A similar analysis, for the EUV jet itself (Fig. 11) displays a similar, striking spike-like event in the $\mathrm{O} \mathrm{V}$ and He I data which coincides with a minimum in the Fe XVI emission. This may be due to obscuration of hotter material behind the jet. The silicon data-points are not showing a coherent story; these measurements are well off the disc 

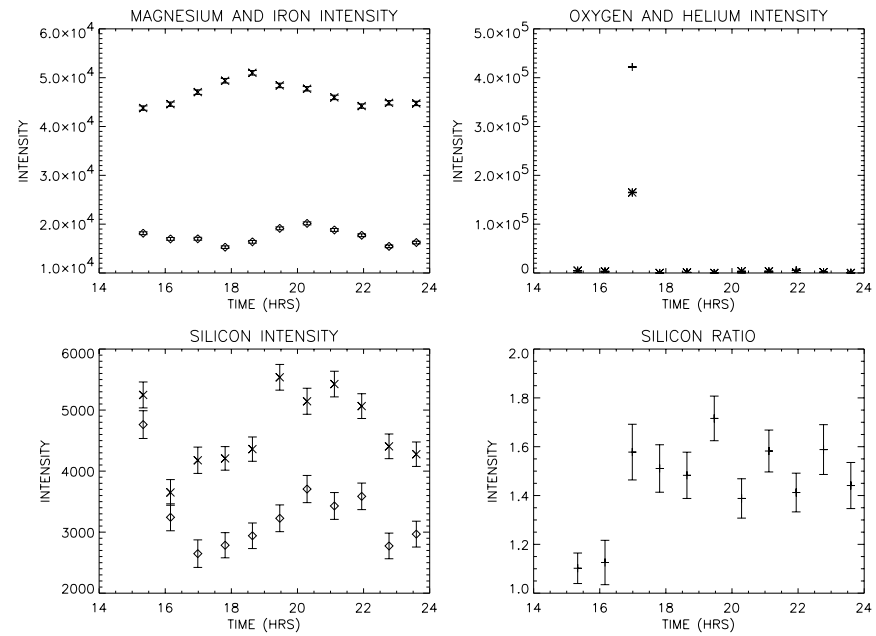

Fig. 10. Time-intensity profiles for the different emission lines from a pixel group occupied by the small loop. For each panel the intensity error bars are shown. The data points are the following, for each panel. Top left: diamonds - Mg IX, crosses - Fe XVI. Top right: asterisks - O V, crosses - He I. Bottom left: crosses - Si X 356, diamonds - Si X 347. Bottom right the ratio Si X 356/347.
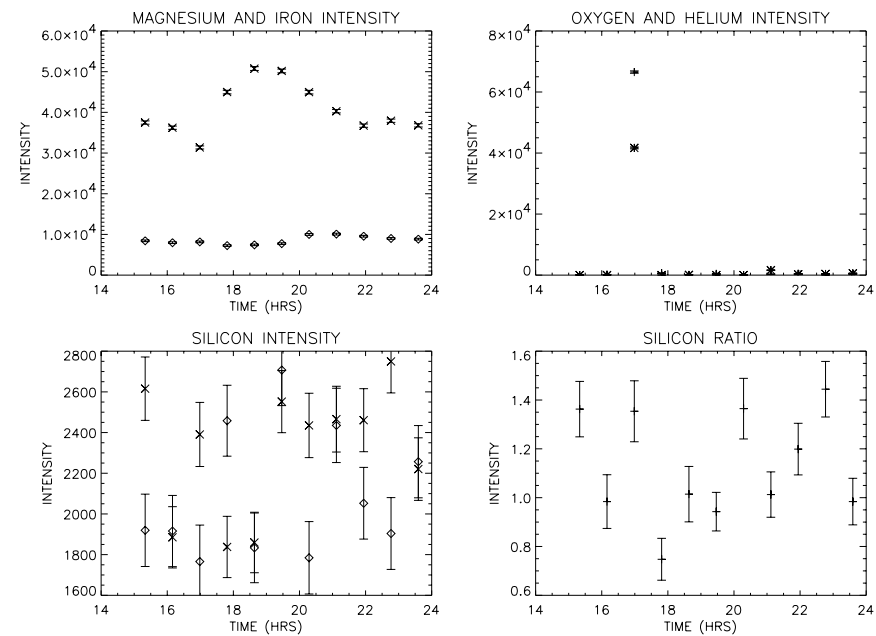

Fig. 11. The same display as Fig. 10, for the EUV jet.

and the error bars are large because of the extremely low intensities.

Figure 12 shows the intensity-time curves for the fanlike event which was detected in the Mg IX data. It is seen as a brightening in the Mg IX profile between 16:00 and 18:00 UT. The Fe XVI data does not show a similar event. Over the same period, the $\mathrm{O} \mathrm{V}$ and He I data display a low intensity and no event. The Si X data, from a slightly hotter emission line than the Mg IX data, do show a similar brightening, and show the same gradual decline. The high ratio, of 1.55 suggests a density of order $6.3 \times 10^{9} \mathrm{~cm}^{-3}$. There is one anomalous data-point just prior to 18:00 UT due to the low intensity of the $356 \AA$ line. We believe this to be artificial.
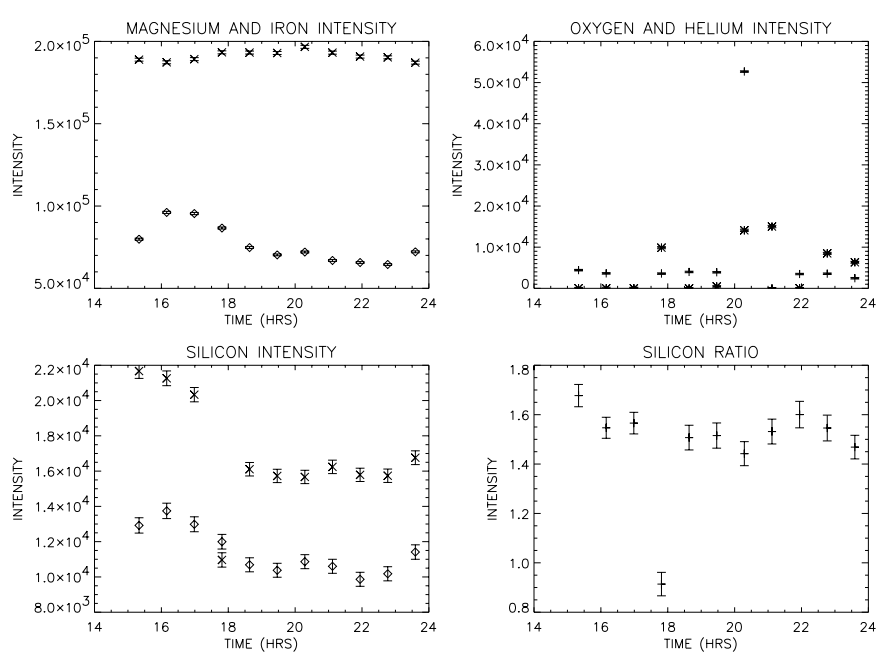

Fig. 12. The same display as Fig. 10, for the Mg IX fan.

\section{Summary}

In the coronagraph data, we see a bright jet of material travelling out at position angle 267 degrees. A projected onset time, assuming no acceleration or deceleration and a zero altitude onset at the limb, is 13:45 UT. Our preferred onset estimate is 15:04 UT; this takes into account the deceleration profile of the jet above the occulting disc. South of the jet is another ejection, namely a small, bright cloud, clearly visible in images from 18:54 UT. This occupies position angles 225 to 245 degrees with a projected onset of 16:30 UT.

The nature of the coronal events of 22 January lead these authors to believe that there is a large CME event in progress. The southern edge is near the south polar region and the site of the jet is at the northern extreme. The small cloud lies in the centre of the CME in this scenario, and could be classed as a "central bright region", possibly associated with a prominence.

The CDS images cover the limb region from 239 to 274 degrees. They show a complex confluence of magnetic loops extending to the north and south of a region which is the source of a bright EUV jet, seen in $\mathrm{O} \mathrm{V}$ and He I data, and a hot fan-like event, detected in $\mathrm{Mg}$ IX data. This region appears to lie at the northern footpoint of the large CME described above, and is the source region of the jet and cloud events seen by LASCO.

The EUV jet is a highly transient event, detected at 16:59 UT. It is only seen in the cooler lines $(250000 \mathrm{~K}$ and $20000 \mathrm{~K}$ ). Doppler analysis shows that it is a spiralling column of plasma displaying plasma acceleration with altitude. Coincident with the EUV jet is the brightening of a small loop at the foot of the jet. Neither the jet nor the loop are visible in earlier or later images. The EUV jet was not visible in the earlier image at 16:09 UT. Rather surprisingly, this confirms that this event was not the onset of the jet seen in the corona. In addition, the EUV jet is at a lower latitude (252 degrees) than the coronal jet, though we do note its non-radial direction. This suggests that either the EUV jet does not result in matter being 
expelled from the corona or the event is part of the ejection process for the coronal jet, but is not part of the initial onset. It seems more plausible to suggest that the EUV jet is associated with the onset of the small cloud in the coronagraph data. It lies at the northern extreme of this cloud and the onset time is consistent.

The fan-like event seen in the Mg IX data was at position angle 255 degrees and was first seen in an image at 16:09 UT. The previous image was at 15:19 UT. The fan is highly non-radial, leaning to the north. Doppler analysis shows no indication of plasma spiralling as with the EUV jet. Given the projected onset of the coronal jet and its position angle, it appears that the $\mathrm{Mg}$ IX fan is the EUV observation of the onset of the LASCO jet.

In summary,

- we believe that there is a large, rather diffuse CME with a jet-like eruption at one footpoint and a bright cloud (central bright region);

- the EUV images/spectra are directed to the northern footpoint of the ejection and allow a detailed analysis of the activity in that region;

- a bright EUV jet, of relatively cool material (less than $250000 \mathrm{~K}$ ), erupts as an adjacent, small loop brightens. The plasma in the jet is accelerating in a spiral structure with altitude. The jet appears to relate to the onset of the small cloud seen later in the outer corona;

- a fan-like event in the million $\mathrm{K} \mathrm{Mg} \mathrm{IX} \mathrm{data} \mathrm{appears}$ to signal the onset of the coronal jet event. This fan lies to the north of the EUV jet and shows no sign of spiral plasma motion.

\section{Interpretation}

In the most general terms, it seems that the sequence of events is the consequence of interactions between the many loops whose footpoints are located within the area viewed by CDS. Whether this is a consequence in itself of loop-loop interactions either driven by the ascending $\mathrm{CME}$, or driving the CME onset, is difficult to determine. Certainly the coincident and sudden brightening of the small EUV loop with the adjacent EUV spiraling jet, suggests that there is a significant interaction between an open and closed magnetic system resulting in the acceleration of matter into the corona.

These EUV jets or sprays appear to relate to, or have common characteristics to other jet-like phenomena observed at different wavelengths, for example, $\mathrm{H} \alpha$ surges or sprays, X-ray jets (detected using the Yohkoh instrumentation) and the EUV coronal hole tornado events reported by Pike \& Mason (1998).

The characteristics of the EUV jet events are in many ways very similar to the jet events reported from Yohkoh observations by Shibata et al. (1996). Their X-ray jets have an average length of $1.5 \times 10^{5} \mathrm{~km}$, i.e. 200 arcsec. The three events of Fig. 1 show that our jet events extend to over 150 arcsec; the actual altitude achieved is not known due to the limited field of view. The average velocity of the Yohkoh jets is given at $200 \mathrm{~km} \mathrm{~s}^{-1}$. The 22 January EUV jet achieved speeds of between 190 and $380 \mathrm{~km} \mathrm{~s}^{-1}$ at the edge of the field of view, and showed clear acceleration. However, we must note that the measured speed is in the line of sight for the CDS data and is measured from apparent motion of bright features in the Yohkoh data. The Yohkoh jet lifetimes range from a few minutes to a few hours. This is consistent with the lifetimes for our EUV jets.

However, apart from the Mg IX/Fe XVI fan-like features shown above for the 22 January event, most of the EUV jets shown here are identified in the $\mathrm{O} \mathrm{V}$ and $\mathrm{He}$ I data, i.e. for temperatures of $250000 \mathrm{~K}$ and $20000 \mathrm{~K}$. These are not events that would be seen by the Yohkoh Soft X-ray Telescope. However, the physical processes leading to the jets may be similar. Indeed, the identification of a bright feature at the base of the EUV jets, and in particular, the small loop at the base of the 22 January jet, is also consistent with the Yohkoh observations.

The speeds identified in the EUV and X-ray data are more consistent with $\mathrm{H} \alpha$ surges (up to a few $100 \mathrm{~km} \mathrm{~s}^{-1}$ ) than the $\mathrm{H} \alpha$ spray events, which display speeds above the solar escape velocity. However, coronagraph data clearly show that at least some of the X-ray/EUV jets are the source of small or narrow ejecta which leave the Sun.

Various jet-like models exist which could be appropriate for the events described here (e.g. Shibata et al. 1996; Canfield et al. 1996). However, many approaches must be ruled out because we do not detect a flare-driven flow and for the main EUV jet event we detect clear rotation, which is not addressed by many models. The Canfield et al. model includes provision for hot and cool plasma motion. They describe the interaction, perhaps due to merging driven by photospheric motion, between a cool $(\mathrm{H} \alpha)$ loop and a group of open field lines. Reconnection occurs between the two magnetic structures. This allows the cool material access to the open fields (seen as the $\mathrm{H} \alpha$ surge) and the reconnection site itself generates the heating and acceleration for the X-ray jet. Thus, the basic structure of loop interacting with open field structure is very similar to the magnetic scenario shown for the 22 January event (Fig. 7 in particular). However, in the Canfield et al. (1996) model, the reconnection releases stored twist in the magnetic fields and this results in a visible untwisting of the magnetic fields which propagates into the jet. This could be the source of the rotating column seen in our case in the $\mathrm{O} \mathrm{V}$ data.

However, this model does not cater for some aspects of the observation. It does not explain the apparent acceleration with altitude which is detected, and there is no clear evidence for blue-red shifted jets at the interaction of the small loop and the base of the jet, i.e. the reconnection site. Also, we do not witness temperatures above 250000 K. Finally, in their picture, the cool material is contained within the small loop and this material is allowed access to the high corona after the reconnection process. In the current data-set, the small loop is not 


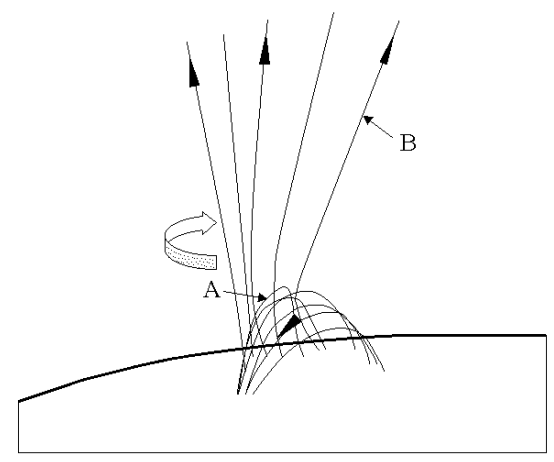

Fig. 13. A schematic model for the EUV jet.

visible in temperatures down to $20000 \mathrm{~K}$ except at the time of the jet event.

We propose that there is magnetic reconnection low in the solar atmosphere as the small loop and open fields interact. The dense, cool plasmas low in the chromosphere and transition region are then given access to the loop and the open fields and thus the loop and the jet are evident at the same time.

We believe that the most appropriate model for the rotating, jet-like events must involve the interaction with a low-lying loop and this picture may well be appropriate for the EUV tornado events, for the Yohkoh jets and the spray events in $\mathrm{H} \alpha$. In fact the basic process can be quite simply explained using a magnetic accretion model (Whitelamb et al. 2001). The model is presented in Fig. 13.

The basic starting point is a confined set of open field lines, possibly located at a confluence of loop systems as in the January 22 event. There may be a fan- like modest outflow of plasma. Indeed, an outflow would drive some rotation in itself, as in the picture due to Spicer \& Sudan (1984). The outflow current will induce a magnetic field perpendicular to the flow of plasma which in itself will drive a helical flow. However, this will not provide a coherent large-scale rotation of a structure of the size of the EUV jet. A basic consideration of the plasma speeds and possible magnetic field strengths reveals spiraling motion with radii well under $1 \mathrm{~km}$, and probably nearer to $100 \mathrm{~m}$. This will be seen as a broadening of the emission lines from the region of the jet and will not provide a coherent large-scale rotation of the feature. For this, we must introduce an asymmetry which can influence the large-scale magnetic structure.

This can be done by the interaction of magnetic structures, through a simple accreting magnetic model. In the case of our model (Fig. 13) it is the interaction of the open field lines and the adjacent closed loop system. One possible scenario is for the small loop to impinge on the open field system. This could drive reconnection as shown in the figure, where the open field line labelled " $\mathrm{B}$ " has reconnected with the closed loop, "A" as the closed system interacts with the open lines. The restructuring of the open loop system produces a kink in the open field line, "B", resulting in a vertical velocity flow and a coherent rotation as indicated by the curved arrow. There is no need for a twisted magnetic structure; simply a requirement for the open field system to encounter an asymmetry which can influence the open field strength. The "accretion" can continue if the loop and open field systems continue to be driven together and this can drive acceleration and continued rotation.

Such a model allows a coherent rotation when an open field system and a closed loop, or similar configurations, interact. Thus, given the nature of the solar atmosphere, we might expect rotating jet-like events to be quite common.

Acknowledgements. CDS was built and is operated by a consortium led by the Rutherford Appleton Laboratory and including the Mullard Space Science Laboratory, the NASA Goddard Space Flight Center, Oslo University and the MaxPlanck-Institute for Extraterrestrial Physics, Garching. SOHO is a mission of international cooperation between ESA and NASA. We would like to thank Dr. Joe Gurman for some extremely valuable comments and advice on this work.

\section{References}

Brueckner, G. E., \& 14 co-authors 1995, Sol. Phys., 162, 357

Canfield, R. C., Reardon, K. P., Leka, K. D., et al. 1996, ApJ, 464, 1016

Delaboudiniere, J.-P., Artzner, G. E., Brunaud, J., \& 25 coauthors 1995, Sol. Phys., 162, 291

Harrison, R. A. 1997, in Proc. 31st ESLAB Symp., ESA SP415,121

Harrison, R. A., \& 38 co-authors 1995, Sol. Phys., 162, 233

Harrison, R. A., \& Lyons, M. 2000, A\&A, 358, 1097

Innes, D. E., Inhester, B., Srivastava, N., et al. 1999, Sol. Phys., 186, 337

Mason, H. E., Young, P. R., Pike, C. D., et al. 1997, Sol. Phys., 170,143

Pike, C. D., \& Mason, H. E. 1998, Sol. Phys., 182, 333

Shibata, K., Shimojo, M., Yokoyama, T., \& Ohyama, M. 1996, Astron. Soc. Pacfic Conf. Ser., 111, 29

Spicer, D. S., \& Sudan, R. N. 1984, ApJ, 280, 448

Whitelam, S., Ashbourn, J. M. A., Bingham, R., Shukla, P. K., \& Spicer, D. S. 2001, A\&A, submitted 